\title{
The Medial Prefrontal Cortex and the Deceptiveness of Memory
}

\author{
Marlieke T.R. van Kesteren and Thackery I. Brown \\ Department of Psychology, Stanford University, Stanford, California 94305 \\ Review of Warren et al.
}

Imagine you have read an interesting article that strongly reminded you of one of your former colleagues' research. You notice that is written by a different research group, but you do not pay too much attention to this and read the article with much pleasure. A few months later you meet your former colleague at a conference and remember the paper you read. You tell him/her that you really enjoyed reading their article. Relating the new article to your colleagues' research has clearly helped you remember its contents, but you have also falsely remembered the article to be theirs. How did that happen?

This situation is one example of how an important process that allows us to integrate and generalize across memories in daily life can also open opportunities for mnemonic errors. The medial prefrontal cortex (mPFC) is thought to be one structure underlying these abilities. In recent years, mPFC function has emerged as a key area of study for understanding higherorder memory and decision-making processes. It is a large, functionally and cytoarchitectonically diverse region, whose intimate connectivity with the medial temporal lobes (MTL) may underlie its involvement in a multitude of memory-

Received July 25, 2014; revised Aug. 21, 2014; accepted Aug. 25, 2014. M.T.R.v.K. is supported by a Rubicon Fellowship from the Netherlands Organization for Scientific Research. We thank Rik Henson for proofreading.

Correspondence should be addressed to Dr. Marlieke T. R. van Kesteren,

Department of Psychology, Jordan Hall, Building 420, Mail Code 2130, Stanford, CA 94305. E-mail: m.vankesteren@stanford.edu.

DOI:10.1523/JNEUROSCI.3091-14.2014

Copyright $(\odot) 2014$ the authors $\quad 0270-6474 / 14 / 3413569-02 \$ 15.00 / 0$ related tasks. Lesions to the $\mathrm{mPFC}$ lead to a range of differences in decision-making, judging emotional and moral states, and task switching. Additionally, patients with mPFC damage often confabulate and lack memory-enhancing effects related to personal social and mnemonic reasoning.

Recent interest in the role that prior knowledge (or a schema) plays in learning and memory has provided a new framework for understanding how components of the mPFC contribute to cognition. Theories of mPFC function centered on schemas propose that this region plays a critical role in guiding decision making and mnemonic processes that interact with previously stored knowledge (van Kesteren et al., 2012; Preston and Eichenbaum, 2013). Consequently, patients with lesions to the $\mathrm{mPFC}$ are assumed to lack guidance of prior knowledge in mnemonic reasoning, memory formation, and decision-making. Indeed, mPFC damage has frequently been associated with cognitive and memory impairments. For example, mPFC lesions abolish memory-enhancing effects such as the self-referencing effect in which self-related memories are better remembered (Philippi et al., 2012) and improved transitive inference of relationships between separately learned associations (Koscik and Tranel, 2012). Moreover, in a navigation setting, mPFC damage has been found to impair the ability to overcome interference from alternative, incorrect navigational memories (Ciaramelli, 2008).

Despite the importance of the mPFC for memory and decision making, $\mathrm{mPFC}$ functions may also lead to overgeneralization and overreliance on prior knowledge, which can underlie false memories or false confidence about details of a memory. Consequently, damage to this region could actually improve memory performance in some circumstances. In a recently published paper, Warren et al. (2014) reasoned that if the mPFC is indeed involved in relating current experience and behavior to prior knowledge, then mnemonic consequences of this mechanism should be absent in lesion patients. If mPFC lesion patients cannot draw on semantic or contextual similarities to make judgments about a memory, they are expected to have fewer false memories that arise because of erroneous attribution to previously learned information, such as in the example where an article is misattributed to a colleague. Paradoxically, this will lead them to benefit from their lesion by encoding a memory trace that is less biased toward previously learned information than their healthy peers.

In psychology, a common way to test false memory is through the DeeseRoediger-McDermott (DRM) paradigm, in which participants learn a set of words with a common theme (such as "cold," "blizzard," "winter"). When subsequently tested, participants often falsely assign a related word (e.g., "snow") to the learned list. Because the mPFC has been suggested to link information to a mnemonic schema, Warren and colleagues (2014) hypothesized that patients with lesions in this region would show less of these misattributed 
false memories in the DRM paradigm. They thus tested memory on the DRM paradigm in a group of mPFC-lesioned patients and compared performance to healthy controls. For free recall of words on the list, they found that the patient group showed a significantly lower amount of false memories, indicating that mPFC lesions indeed keep patients from misattributing related words to the learned list. This decrease in false memories likely relates to a shared mechanistic account that also underlies the diminished memoryenhancing effects described above. Additionally, these findings support direct predictions from the schema literature that hypothesize that $\mathrm{mPFC}$ lesions lower enhancing effects of prior knowledge on learning and consolidating new information. This could then lead to a weaker bias toward knowledge structures and a consequent decrease in false memories at retrieval.

Moving forward, a challenge for researchers seeking to understand the complex functions of the mPFC is to identify the links between empirical data and extant theoretical accounts. In rodents, $\mathrm{mPFC}$ lesions have been shown to abolish the task/context selectivity of some neurons representing objects at locations (Navawongse and Eichenbaum, 2013), potentially impairing the ability to leverage contextual information to retrieve specific memories. Based on these findings and the findings of Warren and colleagues (2014), mPFC functioning might serve as a mechanism for flexibly accessing memories based on contextual information including task rules and goals (Kroes and Fernández, 2012; Brown et al., 2014). The decrease in false alarms found by Warren and colleagues (2014) could then represent a deficit in the natural ability of humans to leverage contextual information (such as the "theme" of the words being tested or your knowledge about your colleagues' research when reading a new paper) to make mnemonic judgments when memory traces are weak. Such a mechanism is typically beneficial when filtering incoming information for relevance or basing decisions on prior outcomes.
The findings of Warren and colleagues (2014) might motivate researchers to take a different view on memory systems and the characterization of memory success. In an experimental setting, whether a memory is remembered or forgotten might not only result from brain states (e.g., attention and motivation) at the moment of encoding, but may also be influenced by complex interactions with prior knowledge. Activation of relevant prior knowledge can help boost new memories and store them more schematically, because they can be directly related to a network of previously learned associations (van Kesteren et al., 2012). When memories lack such a connection to prior knowledge, they may be forgotten more easily or may require stronger retrieval cues to be activated. A critical question in memory research is therefore whether the relationship between newly learned information and prior knowledge can also lead memories to be stored differently: either more episodically detailed or assimilated within previously stored semantic traces.

In addition to encoding processes, offline consolidation processes are also suggested to be affected by interactions with prior knowledge (Tse et al., 2007). Therefore, the role of the MPFC in consolidating knowledge should be further examined in future research. For example, do mPFC lesion patients show retrograde amnesia that is specific to the prior knowledge effects mentioned above? Consequently, will they also show less of the described false memory effects for knowledge that was acquired before their lesion or are the mPFC influences specific to encoding processes? Furthermore, are the decreased false memory effects that Warren and colleagues (2014) describe preserved over a future period of consolidation or do they fade away? And finally, regardless of the answers to the previous questions, what would the findings mean for the interpretation of previously reported retrograde amnesia effects following MTL lesions?

In the real-world example where you falsely remember the authors of a great paper, the fact that you forgot that the paper was actually written by other research- ers might be a consequence of your brain trying to make the complex world more consistent and predictable. Consequently, less significance is given to the fact that the data actually originate from a different and unknown research group, which leads to misattribution errors. The findings of Warren and colleagues (2014) suggest that the mPFC may play a role in such errors, and provide important insights into the conditions under which $\mathrm{mPFC}$ function may impair, rather than facilitate, certain types of memory.

\section{References}

Brown TI, Whiteman AS, Aselcioglu I, Stern CE (2014) Structural differences in hippocampal and prefrontal gray matter volume support flexible context-dependent navigation ability. J Neurosci 34:2314-2320. CrossRef Medline

Ciaramelli E (2008) The role of ventromedial prefrontal cortex in navigation: a case of impaired wayfinding and rehabilitation. Neuropsychologia 46:2099-2105. CrossRef Medline

Koscik TR, Tranel D (2012) The human ventromedial prefrontal cortex is critical for transitive inference. J Cogn Neurosci 24:1191-1204. CrossRef Medline

Kroes MC, Fernández G (2012) Dynamic neural systems enable adaptive, flexible memories. Neurosci Biobehav Rev 36:1646-1666. CrossRef Medline

Navawongse R, Eichenbaum H (2013) Distinct pathways for rule-based retrieval and spatial mapping of memory representations in hippocampal neurons. J Neurosci 33:1002-1013. CrossRef Medline

Philippi CL, Duff MC, Denburg NL, Tranel D, Rudrauf D (2012) Medial PFC damage abolishes the self-reference effect. J Cogn Neurosci 24:475-481. CrossRef Medline

Preston AR, Eichenbaum H (2013) Interplay of hippocampus and prefrontal cortex in memory. Curr Biol 23:R764-R773. CrossRef Medline

Tse D, Langston RF, Kakeyama M, Bethus I, Spooner PA, Wood ER, Witter MP, Morris RG (2007) Schemas and memory consolidation. Science 316:76-82. CrossRef Medline

van Kesteren MT, Ruiter DJ, Fernández G, Henson RN (2012) How schema and novelty augment memory formation. Trends Neurosci 35:211-219. CrossRef Medline

Warren DE, Jones SH, Duff MC, Tranel D (2014) False recall is reduced by damage to the ventromedial prefrontal cortex: implications for understanding the neural correlates of schematic memory. J Neurosci 34:7677-7682. CrossRef Medline 